\title{
ALIMENTAÇÃO, ANEMIA E DESNUTRIÇÃO EM CRIANÇAS EM FASE PRÉ-ESCOLAR: UMA REVISÃO
}

Daniela Bergamini Teixeira, Bárbara Pereira da Silva, Maria Eliza de Castro Moreira. Alimentação, anemia e desnutrição. Revista Saúde Dinâmica, vol. 3, núm.1, 2020. Faculdade Dinâmica do Vale do Piranga. 


\title{
Alimentação, anemia e desnutrição em crianças em fase pré-escolar: uma revisão
}

\author{
Food intake, anemia, and malnutrition in preschool children: a review
}

Daniela Bergamini Teixeira ${ }^{l}$, Bárbara Pereira da Silva ${ }^{l}$, Maria Eliza de Castro Moreira ${ }^{l}$

${ }^{I}$ Faculdade Dinâmica do Vale do Piranga - Rua G, 205 - Bairro Paraíso - Ponte Nova - MG - Cep: 35430-302

\section{Resumo}

O presente artigo traz uma revisão de literatura com o objetivo de avaliar o consumo alimentar, a presença de anemia e desnutrição em pré-escolares assim como a associação entre esses fatores. A pesquisa foi realizada na base de dado eletrônica, Scopus, nos quais foram selecionados artigos originais, publicados entre os anos 2010 a 2019, em inglês e português, sendo utilizado os descritores de assunto "Alimentação Escolar_Desnutrição", "Alimentação Escolar_Anemia", "School_Feeding-and-Pre-School-Anemia", "School_Feeding-and-Pre-School-Malnutrition". Foram encontrados 591 artigos e destes 9 foram selecionados a partir da leitura do resumo/artigo completo. Os artigos selecionados eram do Brasil ou de países da África. Por meio da leitura dos artigos pode-se concluir que apesar das políticas públicas implementadas nos países, principalmente nos países mais pobres, ainda é relevante o número de crianças em fase pré-escolar que apresentam deficiências nutricionais como anemia e desnutrição. Os fatores socioeconômicos como renda familiar, grau de escolaridade, saneamento básico, dentre outros são responsáveis por grande parte destas deficiências.

Palavras-chave: Alimentação do pré-escolar, anemia ferropriva, deficiência nutricional do préescolar

\begin{abstract}
This paper presents a literature review that aimed to analyze food intake, presence of anemia and malnutrition in preschoolers as well as the association between these factors. The research was conducted in electronic database, Scopus, in which were selected original papers, published between the years 2010 and 2019, in english and portuguese, using the subject descriptors "School Feeding_Malnutrition", "School Feeding_Anaemia", "School_Feeding-and-Pre-School-Anemia", "School_Feeding-and-Pre-SchoolMalnutrition". We founded 591 papers and of these 9 were selected from the reading of the abstract/full paper. The articles selected were from Brazil or from African countries. Due to reading of the papers it was concluded that despite the public policies implemented in the countries, especially in the poorer countries, the number of preschool children with nutritional deficiencies such as anemia and malnutrition is still relevant. Socioeconomic factors such as family income, education level, basic sanitation, among others are responsible for most of these deficiencies.
\end{abstract}

Keywords: Preschool feeding, anaemia, nutritional deficiency of preschoolers. 


\section{INTRODUÇÃO}

As escolas têm a função de cuidar e educar, atingindo a ideia de que o direito aos cuidados básicos devam garantir o bem estar de todas as crianças. $\mathrm{O}$ aumento da procura por lugares de qualidade, que irão proporcionar os benefícios necessários para o desenvolvimento da criança é recorrente; porém, a vigilância das normas que regulamentam o funcionamento desses ambientes bem como o número de profissionais capacitados para o exercício da função não atende à demanda que existe atualmente no Brasil (PEREIRA et al., 2010).

É durante o período pré-escolar que as crianças constroem hábitos alimentares, que, se não for devidamente supervisionado, pode acarretar em distúrbios nutricionais. As crianças representam um grupo muito vulnerável a deficiência nutricional devido ao crescimento rápido e a imaturidade fisiológica e imunológica (LIMA et al., 2011). O crescente risco do desenvolvimento de doenças na fase pré-escolar faz com que a introdução de hábitos alimentares saudáveis se torne necessário, pois, além da manutenção das funções vitais, o organismo da criança precisa de nutrientes para o crescimento, desenvolvimento da estrutura óssea e do sistema nervoso. Assim, a falta ou o excesso de alimentos poderão desencadear diferentes doenças (BRASIL, 2014).

Nas últimas décadas, o Brasil reduziu significativamente a taxa de desnutrição crônica entre menores de 5 anos (PEREIRA et al., 2017). Porém, essa doença ainda continua sendo um problema de saúde pública, principalmente em populações de baixa renda, onde as mães possuem baixa escolaridade além de não têm acesso a informações sobre meios para obter uma dieta equilibrada para seus filhos (PEREIRA et al., 2017).

Embora a amamentação possa salvar vidas e evitar a desnutrição, por exemplo, apenas $42 \%$ das crianças menores 6 meses de idade são amamentadas exclusivamente e um número crescente de bebês é alimentado com fórmula infantil (BRASIL, 2019). De acordo com Vieira, Souza, e Cervato-Mancuso (2010) é necessário avaliar o estado nutricional das crianças, pois a desnutrição infantil pode levar a várias complicações na fase adulta.

Estudos sobre o consumo alimentar de pré-escolares atestam baixo consumo de ferro, cálcio, vitamina A e fibras (GOULART et al., 2010, DELLA LUCIA et al., 2016). Os principais prejuízos dessas carências são: alterações na imunidade da criança, maior susceptibilidade a infecções e funcionamento inadequado do metabolismo (RODRIGUES et 
al., 2011). Dentre as várias doenças que podem acometer as crianças, pode-se citar a anemia ferropriva (ANOKYE et al., 2018). A anemia tem uma prevalência alta em todas as faixas etárias e no Brasil está presente tanto em populações carentes quanto populações de maior poder aquisitivo (DELLA LUCIA et al., 2016). Entre os grupos de risco, pode-se citar as crianças em idade pré-escolar, uma vez que a necessidade nutricional desse mineral é elevada (BRASIL, 2010). Cerca de 60,2\% das crianças brasileiras menores de cinco anos apresentam anemia por deficiência de ferro (Brasil, 2009a). Diante do exposto, o objetivo deste trabalho foi o avaliar o consumo alimentar, a presença de anemia e de desnutrição em pré-escolares, assim como a associação entre esses fatores.

\section{MATERIAIS E MÉTODOS}

Trata-se de uma revisão de literatura, realizada entre agosto e setembro de 2019, que utilizou bases de dados eletrônicas (Scopus), nos quais foram selecionados artigos originais, publicados entre os anos 2010 a 2019, em inglês e português, sendo utilizado os descritores de assunto "Alimentação Escolar_Desnutrição ou Alimentação Escolar_Anemia ou School_Feeding-and-Pre-School-Anemia ou School_Feeding-and-Pre-School-Malnutrition na base de dados.

Pode-se citar como critério de inclusão os estudos com crianças com idade entre 2 e 5 anos (pré-escolar); estudos originais provenientes de pesquisas quantitativas ou qualitativas, que abordaram alimentação do pré-escolar, desnutrição e anemia nesta faixa etária; Os critérios de exclusão utilizados foram: estudos com crianças menores de 2 anos e maiores de 6 anos; artigos de revisão da literatura, artigos em duplicidade, artigos de opinião, editoriais e publicações que não discorreram especificamente sobre o assunto pretendido. Além disso, foram excluídos também os artigos que discorriam sobre políticas públicas sobre alimentação do escolar. 
Tabela 1: Artigos incluídos na revisão sistemática

\begin{tabular}{|c|c|c|c|c|c|}
\hline Autor & Grupo Amostral & $\begin{array}{l}\text { Tipo de } \\
\text { Estudo }\end{array}$ & $\begin{array}{l}\text { Local de } \\
\text { Estudo }\end{array}$ & $\begin{array}{l}\text { Metodologia } \\
\text { Utilizada }\end{array}$ & Objetivo \\
\hline $\begin{array}{l}\text { Houghton et } \\
\text { al.,2019 }\end{array}$ & 500 pré-escolares & Transversal & Quênia & $\begin{array}{l}\text { Questionário sócio } \\
\text { demográfico e de } \\
\text { saúde }\end{array}$ & $\begin{array}{l}\text { Investigar o estado de micronutrientes e saúde de } \\
\text { pré-escolares }\end{array}$ \\
\hline $\begin{array}{l}\text { Anokye, et } \\
\text { al., } 2018\end{array}$ & $\begin{array}{l}228 \text { crianças } \\
\text { menores de } 5 \\
\text { anos }\end{array}$ & Transversal & Gana & $\begin{array}{l}\text { Questionário } \\
\text { estruturado }\end{array}$ & $\begin{array}{l}\text { Determinar a causas, sinais e sintomas percebidos, } \\
\text { bem como prevenção da anemia infantil entre } \\
\text { mães de crianças menores de } 5 \text { anos }\end{array}$ \\
\hline $\begin{array}{l}\text { Ewusie et al., } \\
2016\end{array}$ & $\begin{array}{l}2379 \text { crianças } \\
\text { menores de } 5 \\
\text { anos }\end{array}$ & Populacional & Gana & $\begin{array}{l}\text { Questionário sócio } \\
\text { demográfico }\end{array}$ & $\begin{array}{l}\text { Identificar os fatores de risco associados à } \\
\text { desnutrição infantil. }\end{array}$ \\
\hline $\begin{array}{l}\text { Akodu et al., } \\
2016\end{array}$ & $\begin{array}{l}89 \text { crianças } \\
\text { menores de } 5 \\
\text { anos }\end{array}$ & Transversal & Nigéria & $\begin{array}{l}\text { Coleta de amostras de } \\
\text { sangue }\end{array}$ & $\begin{array}{l}\text { Estimar a prevalência e fatores associados a } \\
\text { anemia ferropriva em menores de } 5 \text { anos }\end{array}$ \\
\hline $\begin{array}{l}\text { Al Rifai et al., } \\
2015\end{array}$ & $\begin{array}{l}3789 \text { pré- } \\
\text { escolares }\end{array}$ & Transversal & Jordânia & $\begin{array}{l}\text { Questionário sócio } \\
\text { demográfico e de } \\
\text { saúde }\end{array}$ & $\begin{array}{l}\text { Examinar mudanças na prevalência de anemia e } \\
\text { seus correlatos entre crianças em idade pré- } \\
\text { escolar após implementação da fortificação da } \\
\text { farinha de trigo com vários micronutrientes. }\end{array}$ \\
\hline $\begin{array}{l}\text { Della Lucia et } \\
\text { al., } 2015\end{array}$ & 99 pré-escolares & $\begin{array}{l}\text { Estudo de } \\
\text { intervenção }\end{array}$ & Brasil & Consumo alimentar & $\begin{array}{l}\text { Avaliar a necessidade de inclusão de arroz } \\
\text { fortificado nas refeições de pré-escolares, } \\
\text { considerando a ingestão de alimentos, a qualidade } \\
\text { de sua dieta e o impacto de seu uso as medições } \\
\text { laboratoriais das crianças }\end{array}$ \\
\hline
\end{tabular}




\begin{tabular}{|c|c|c|c|c|c|}
\hline $\begin{array}{l}\text { Longo-Silva } \\
\text { et al., } 2014\end{array}$ & $\begin{array}{l}366 \text { crianças entre } \\
12 \text { e } 36 \text { meses }\end{array}$ & $\begin{array}{l}\text { Estudo } \\
\text { transversal } \\
\text { descritivo }\end{array}$ & Brasil & $\begin{array}{l}\text { Pesagem direta dos } \\
\quad \text { alimentos }\end{array}$ & $\begin{array}{l}\text { Avaliar a ingestão de cálcio, proteína e sódio e } \\
\text { compará-la com a recomendada, em crianças } \\
\text { que frequentam creches públicas. }\end{array}$ \\
\hline $\begin{array}{l}\text { Veiga et al., } \\
2010\end{array}$ & $\begin{array}{l}80 \text { pré-escolares, } \\
\text { entre } 12 \text { e } 71 \\
\text { meses }\end{array}$ & $\begin{array}{c}\text { Estudo } \\
\text { transversal }\end{array}$ & Brasil & $\begin{array}{l}\text { Questionário } \\
\text { socioeconômico, } \\
\text { demográfico e } \\
\text { ambiental }\end{array}$ & $\begin{array}{l}\text { Determinar os perfis bioquímicos de crianças } \\
\text { desnutridas cronicamente }\end{array}$ \\
\hline $\begin{array}{l}\text { Pereira et al., } \\
2013\end{array}$ & 51 pré-escolares & $\begin{array}{l}\text { Estudo } \\
\text { longitudinal }\end{array}$ & Brasil & $\begin{array}{l}\text { Pesagem direta dos } \\
\text { alimentos e registro } \\
\text { alimentar }\end{array}$ & $\begin{array}{l}\text { Monitorar o estado nutricional de pré-escolares } \\
\text { de uma creche pública }\end{array}$ \\
\hline
\end{tabular}


A primeira etapa de seleção das produções foi realizada mediante a leitura e a análise dos títulos e resumos de todos os artigos identificados. Já na segunda etapa, foi determinada pela leitura na íntegra dos estudos selecionados, a qual possibilitou que outros textos também fossem excluídos por não atenderem à proposta da revisão. Na tabela se encontra as análises descritivas dos estudos selecionados, com autor, data da publicação e a abordagem principal de cada artigo (Tabela 1).

\section{RESULTADOS E DISCUSSÃO}

Foram encontrados 591 artigos na base do Scopus. Desses, 62 artigos foram selecionados com base na leitura de títulos e/ou resumos. Dentre os artigos excluídos pois estavam em duplicidade ou se tratavam de revisão bibliográfica ou que não abordavam o assunto.

Dentre os 62 artigos selecionados com base de dados de leitura do título, 21 foram selecionados a partir da leitura do resumo dos mesmos. Sendo que 41 foram excluídos pelos seguintes motivos: 9 artigos se tratavam de revisão bibliográfica e 32 artigos não enquadravam no tema, onde abordavam outros assuntos de alimentação que não fosse o assunto em questão, não se referiam a pré-escolares ou fugiam completamente do assunto de interesse (Figura 1). Dos 21 artigos selecionados, 12 artigos foram excluídos após leitura completa dos mesmos, sendo 6 artigos indisponíveis gratuitamente, 5 fugiam do tema abordado e 1 artigo em duplicidade. 


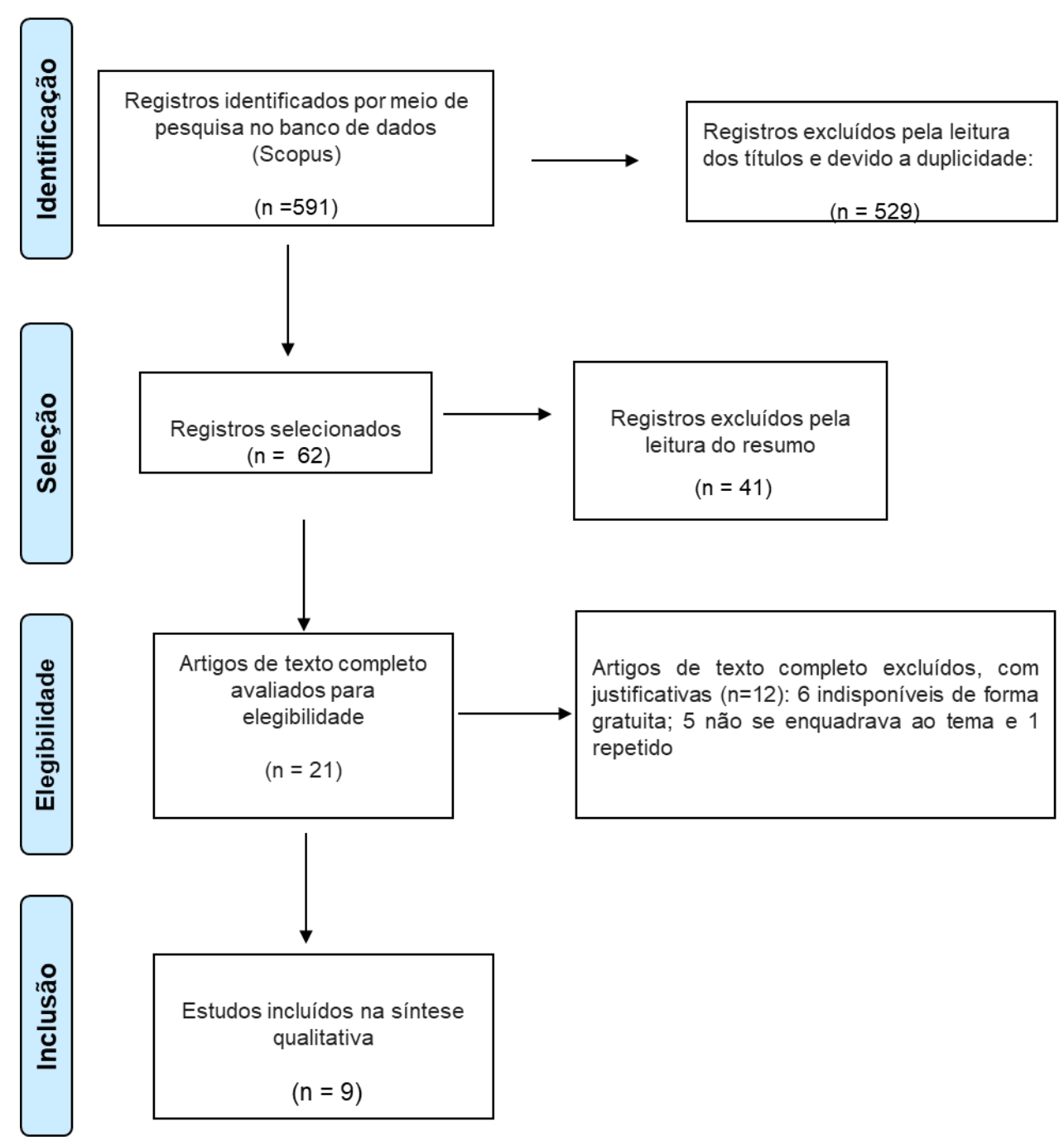

Figura 1. Fluxograma do processo da análise e seleção dos artigos utilizados na revisão.

Quanto às características gerais, a publicação mais antiga era de 2010, sendo que a maioria dos artigos foram originados do Brasil. Na avaliação dos artigos foram encontrados diversos tipos de ferramentas utilizadas para avaliar o consumo alimentar, desde entrevistas, pesquisas populacionais, questionários até diários alimentares. O grupo amostral utilizado nos estudos divergiu quanto ao número. Na Tabela 2 encontra-se uma síntese dos dados disponíveis nos artigos incluídos na revisão.

Dentre os artigos selecionados três abordaram sobre a prevalência de anemia, dois relacionaram a anemia e a desnutrição juntos, um outro associou a anemia a más práticas alimentares, um artigo discorreu sobre a prevalência de desnutrição e um último abordou o baixo consumo alimentar de micro e macronutrientes. 
Tabela 2. Síntese dos artigos incluídos na revisão

Autor

Houghton et al., 2019
- Prevalência de anemia e a deficiência de ferro: maior em crianças Maasai.

- Não foram observadas diferenças na prevalência de deficiências de zinco, selênio, vitamina A ou vitamina D entre as tribos.

- Ferro corporal, PCR e idade são preditores das concentrações de hemoglobina para ambas as tribos.

Anokye et al., 2018

- Anemia é causada por más práticas alimentares (43\%) e febre (37\%).

- Esse fato pode ser evitado: nutrição adequada (23\%), desparasitação regular (19\%) e do reabastecimento exclusivo (25\%).

- Escolaridade das mães e o número de filhos se associam à percepção sobre a anemia.

- 35,6\% das crianças apresentam desnutrição.

Ewusie et al., 2016

- Sexo e idade da criança, estado educacional e nutricional da mãe e o status financeiro da família: fatores de riscos para a desnutrição infantil

- Necessário realizar ações de educação alimentar e nutricional e práticas de alimentação infantil.

Akodu et al., 2016

-Prevalência de anemia ferropriva foi maior entre as crianças com peso por idade e peso por estatura abaixo de dois escores $\mathrm{Z}$

- A prevalência geral de anemia ferropriva entre as crianças do estudo foi de $10,11 \%$

Al Rifai et al., 2015

- Prevalência de anemia em crianças em idade pré-escolar diminuiu de 40,4\% em 2007 para 33,9\% em 2009

- Redução mais acentuada: crianças menores de 24 meses, crianças vivendo em áreas urbanas, crianças de famílias ricas, crianças que nunca foram amamentadas e crianças bem nutridas.

- Arroz fortificado: melhorou os níveis de zinco, tiamina, ácido fólico e hemoglobina das crianças.

- Inadequação de tiamina, ácido fólico e ferro: menores entre pré-escolares da pré-escola de teste.

Della Lucia et al., 2016

- O estudo demonstrou a eficácia do uso do arroz fortificado, sendo que esse pode ser uma estratégia interessante nos programas de alimentação escolar. 
- Ingestão de sódio ultrapassou a recomendação.

- Relação cálcio/proteína de $11,7 \mathrm{mg} / \mathrm{g}$ foi inferior à adequação $(20 \mathrm{mg} / \mathrm{g})$.

- Falta de abastecimento de água tratada é associada ao grau de desnutrição.

- Anemia foi prevalente entre os desnutridos graves.

Veiga et al., 2010

- Níveis de IGF-1 diminuíram com o aumento da gravidade da desnutrição.

- Maioria das crianças possuem dislipidemia

- Desnutrição crônica provoca alterações endócrinas que ocasionam alterações no perfil metabólico de crianças em idade préescolar.

- Redução de prevalência de E/I, P/I e de anemia.

- Aumento do consumo de energia, carboidratos, proteínas, vitaminas A e C e ferro.

- Importante: monitoramento do estado nutricional de crianças pré-escolares atendidas em creches públicas, bem como ações de educação nutricional.

Pereira et al., 2013

semelhach a insulina tipo 1 ,

IGF: Fator de crescimento semelhante à insulina tipo 1, E/I: estatura para idade; P/I: peso para idade. 


\section{DISCUSSÃO}

No presente trabalho foi realizada uma revisão de artigos sobre alimentação e nutrição de pré-escolares e a incidência de anemia e desnutrição nesta faixa etária. A alimentação infantil é um tema que desperta grande interesse dos pesquisadores, uma vez que essa fase da vida é impulsionada por um período de crescimento constante e de formação de hábitos alimentares. Hábitos alimentares saudáveis durante esse período da vida garante tanto o crescimento físico assim como o desenvolvimento mental, ao mesmo tempo que previne algumas doenças (CARVALHO, 2016).

A adequação da ingestão alimentar é de grande importância, pois é considerado o primeiro passo para a conscientização sobre a ingestão recomendada para a população infantil, especialmente no contexto de creches públicas. O consumo adequado de nutrientes é capaz de prevenir o desenvolvimento de doenças relacionadas à má nutrição como, por exemplo, a desnutrição, anemia, osteoporose, hipertensão e doenças cardiovasculares, em crianças (SILVA et al., 2013).

Neste sentido, o Ministério da Educação criou o Programa Nacional de Alimentação Escolar (PNAE) que é uma iniciativa da política pública brasileira destinada ao ambiente escolar, tendo como foco principal a nutrição suplementar e a educação nutricional de crianças e adolescentes (BRASIL, 2019). A educação alimentar e nutricional no processo de ensino e aprendizagem aborda o tema alimentação e nutrição e o desenvolvimento de práticas saudáveis de vida, na perspectiva da segurança alimentar e nutricional (BRASIL, 2009). A alimentação escolar visa a suprir, no mínimo, $15 \%$ das necessidades nutricionais dos escolares durante sua permanência na escola. Desde de 2006, o Fundo Nacional de Desenvolvimento da Educação (FNDE) determina que os alimentos distribuídos para as escolas devam ser diversificados e advir da agricultura familiar, e que boa parte (70\%) dos recursos do PNAE deve ser utilizado na aquisição de produtos básicos (BRASIL, 2006).

O consumo alimentar entre crianças ainda apresenta resultados bastante controversos. Alguns autores sugerem que, com a utilização dos alimentos advindos da agricultura familiar, a qualidade dos cardápios oferecidos aos pré-escolares melhorou significativamente pois os alimentos in natura são ótimas fontes de proteínas, vitaminas e minerais, fibras e compostos antioxidantes (GONÇALVES et al., 2015). De acordo com outros autores, o consumo diário 
de duas ou três refeições escolares está associado ao menor consumo de produtos ultra processados e a maior participação de alimentos in natura e minimamente processados na dieta dos alunos, em comparação com aqueles que não consomem refeições escolares. Além disso, os estudantes que permanecem em período integral na escola apresentam um melhor estado nutricional do que os estudantes que não consomem refeições escolares ou que permanecem na escola em período parcial (BENTO et al., 2017). Contudo, outros trabalhos nos mostram que o consumo de energia, proteína e cálcio ainda está abaixo do recomendado entre os pré-escolares de creches públicas, sendo que a ingestão de sódio é até três vezes superior à recomendação. Dessa forma, pode-se concluir que o consumo alimentar ainda é inadequado em crianças inseridas no contexto de creches públicas (SILVA-LONGO et al., 2014).

O consumo alimentar está diretamente relacionado ao estado nutricional dos préescolares. Pereira et al. (2013) observou uma redução de prevalência para déficit estatura/idade e peso/idade, além de redução da prevalência de anemia, aumento do consumo de energia, carboidratos, proteínas, vitaminas A e C e ferro. Assim, torna-se uma estratégia muito importante realizar o monitoramento do estado nutricional de crianças pré-escolares atendidas em creches públicas.

Mesmo com estas ações tomadas pelo governo, a desnutrição ainda continua sendo um dos principais desafios da saúde pública, principalmente nos países em desenvolvimento. Ewusier et al., (2016) concluíram que é necessário aumentar a conscientização e melhorar as intervenções direcionadas, incluindo ações de educação alimentar e nutricional e práticas de alimentação infantil. Os principais fatores de risco para a desnutrição podem ser a amamentação exclusiva inferior ao recomendado pela OMS (6 meses) e deficiências de micronutrientes (vitamina A, ferro e zinco). Vale destacar ainda que a desnutrição é responsável por mais de um terço de todas as mortes de menores de cinco anos (BHUTTA, SALAM \& DAS, 2013).

A prevalência de desnutrição em crianças menores de 5 anos geralmente é analisada por idade, região, local de residência, educação da mãe, aleitamento materno exclusivo, momento de introdução alimentação complementar, bem como a quantidade, frequência, consistência e qualidade correta dos alimentos a serem oferecidos à criança, dentre outros fatores sócio demográficos que são identificados como fatores de risco para desnutrição infantil (EWUSIER et al., 2016). Veiga et al., (2010) concluiu que a desnutrição está claramente 
associada a falta de água tradada e que a maioria das crianças desnutridas possuíam anemia e presença de parasitas. $O$ autor relata ainda que quase todas as crianças apresentavam dislipidemia, enquanto baixos níveis de lipoproteína de alta densidade estavam associados ao grau de desnutrição. Além disso, é discutido a relação entre desnutrição crônica e alterações endócrinas, uma vez que essas ocasionam alterações no perfil metabólico de crianças em idade pré-escolar (VEIGAS et al., 2010).

A deficiência de ferro é o distúrbio nutricional mais prevalente no mundo. A prevalência de anemia por deficiência de ferro é de cerca de 47,4\% em crianças pré-escolares, sendo considerada um problema de saúde pública que afeta países desenvolvidos e em desenvolvimento (VALENCIA et al., 2019). Dentre as populações de risco para a anemia ferropriva, as crianças pré-escolares são o grupo mais vulnerável. A anemia infantil tem grandes consequências adversas para a saúde e para o desenvolvimento, incluindo retardo no crescimento, infecções, aumento da morbimortalidade e comprometimento do desenvolvimento motor e cognitivo (GHASSEMI, 2014; SACHDEV, 2013), que podem durar após o tratamento medicamentoso (MANDAL, SAHI, 2016). Corroborando com estes dados, Akodu, Disu, Njokanma, Kehinde (2016) concluíram que existe associação entre crianças que apresentam baixo peso por idade e por estatura a anemia por deficiência de ferro. Entretanto, Anokye et al., (2018), concluiu que a anemia pode ocorrer devido a práticas inadequadas de alimentação e pode ser evitada com uma nutrição adequada e desparasitação. Segundo os autores, crianças anêmicas devem receber tratamento precoce e manter a amamentação exclusiva até os seis meses. Neste estudo eles citam que o número de pessoas nos domicílios, a renda familiar, o nível de educação dos pais, o local de residência e instalações habitacionais, como acesso a água tratada e bens de consumo são fatores que interferem diretamente no desenvolvimento da doença (ANOKYE et al., 2018).

Para diminuir a prevalência de anemia, o Ministério da Saúde vem realizando várias medidas de alcance populacional, como a obrigatoriedade da adição de ferro e ácido fólico às farinhas de milho e trigo (BRASIL, 2017). Além disso, a fortificação e biofortificação de alguns alimentos utilizados no cotidiano da população brasileira, como o arroz, batata doce, mandioca e feijão por exemplo, pode ser uma ótima estratégia para aumentar o aporte de nutrientes ao organismo, principalmente minerais e vitaminas. Segundo Della Lucia et al., (2015) a biofortificação do arroz é uma estratégia interessante nos programas de alimentação escolar para tentar suprir a demanda de ferro nesta faixa etária. Corroborando com este 
estudo, Al Rifai, Nakamura \& Seino (2015), mostraram que a prevalência de anemia em crianças em idade pré-escolar de 6 a 59 meses reduziu, após a implementação da fortificação de grãos de trigo com múltiplos micronutrientes. O declínio foi mais pronunciado em crianças com melhor condição socioeconômico e estado nutricional. Já Houghton et al., (2019) realizou um estudo comparativo entre crianças do Maasai e Kamba para verificar a prevalência de deficiência de ferro e concluiu que as crianças do Maasai apresentaram maior prevalência de anemia, provavelmente devido ao maior consumo de leite de vaca (menor biodisponibilidade de ferro) em detrimento de refeições fortificadas com micronutrientes, bem como à presença de inflamação crônica e danos intestinais.

O estudo apresenta pontos fortes e algumas limitações. Dentre os pontos fortes, pode-se citar os critérios de inclusão e exclusão adotados. Contudo, deve-se destacar como limitação do estudo a grande variabilidade da amostra, o número amostral diversificado e o uso limitado de bases de dados existentes.

\section{CONCLUSÃO}

Diante dos resultados analisados pode-se concluir que ainda é relevante o número de crianças em fase pré-escolar que apresentam deficiências nutricionais, como a desnutrição. Esse fato é evidenciado tanto em países subdesenvolvidos como nos países em desenvolvimento e desenvolvidos. Alguns fatores estão diretamente relacionados a deficiência do estado nutricional das crianças como renda familiar, grau de escolaridade materna, presença de saneamento básico, dentre outros. Além disso, percebe-se que o consumo alimentar inadequado, observado frente ao consumo de carboidratos simples, gorduras e sódio vem aumentando em certas regiões, culminando com o desenvolvimento de sobrepeso e obesidade. Associado a esses fatores, pode-se ainda destacar a redução do consumo de micronutrientes, dentre ele o ferro, o que leva a prejuízos no crescimento e desenvolvimento infantil. 


\section{REFERÊNCIAS}

AKODU, O.S., DISU, E.A., NJOKANMA, O.F., KEHINDE, O.A. Iron deficiency anaemia among apparently healthy pre-school children in Lagos, Nigeria. African Health Sciences, 2016, 16 (1), 61-68.

AL RIFAI, R., NAKAMURA, K., SEINO, K. Decline in the prevalence of anaemia among children of pre-school age after implementation of wheat flour fortification with multiple micronutrients in Jordan. Health Nutrition, 2016, 19 (8), p. 1486-1497.

ANOKYE, R., ACHEAMPONG, E., EDUSEI, A.K., MPRAH, W.K., OFORI-AMOAH, J., AMOAH, V.M.K., ARKORFUL, V.E. Perception of childhood anaemia among mothers in Kumasi: A quantitative approach. Italian Journal of Pediatrics, 2018, 44 (1).

BHUTTA, Z.A., SALAM, R.A., DAS, J.K. Meeting the challenges of micronutrient malnutrition in the developing world. British Medical Bulletin, 2013, 106 (1), p. 7-17.

BRASIL - Fundo Nacional de Desenvolvimento da Educação. Estabelece as normas para a execução do Programa Nacional de Alimentação Escolar. Brasília. Diário Oficial da União, $2006 . \quad$ Disponível em: http://www.fnde.gov.br/fnde/legislacao/resolucoes/item/3106resolu\%C3\%A7\%C3\%A3ocdfnde-n\%C2\%BA-32-de-10-de-agosto-de-2006. Acesso em: 15 outubro 2019.

BRASIL. Fundo das Nações Unidas para a Infância (UNICEF), Má alimentação prejudica a saúde de milhões de crianças em todo o mundo. 2018. Disponível em: https://www.unicef.org/brazil/desnutricao. Acesso em: 22 out.2019.

BRASIL. Ministério da Saúde. Centro Brasileiro de Análise e Planejamento. Pesquisa Nacional de Demografia e Saúde da Criança e da Mulher PNDS, 2006. Série G. Estatística e Informação em Saúde. Brasília. DF, 2009a. 296 p.

BRASIL. Ministério da Saúde. Distrito Federal. Caderno de Atenção Básica. 2010, p. 23-26.

BRASIL. Ministério da Saúde. Secretaria de Atenção à Saúde. Departamento de Atenção Básica. Guia alimentar para a população brasileira, 2. ed., Brasília: Ministério da Saúde, 2014, p. 156.

BRASIL - Presidência da República. Lei no 11.947, de 16 de junho de 2009. Dispõe sobre o atendimento da alimentação escolar e do Programa Dinheiro Direto na Escola aos alunos da educação básica. Brasília: Diário Oficial da União, 2009 b.

BRASIL. Ministério da Saúde. Agência Nacional de Vigilância Sanitária. Resolução RDC no 150 de 13 de Abril de 2017. Aprova o Regulamento Técnico para o enriquecimento das farinhas de trigo e de milho com ferro e ácido fólico. Diário Oficial da República Federativa do Brasil. Brasília, 2017. 
CARVALHO, A.P.L.S. Importância da alimentação para melhorias na aprendizagem de crianças em unidades públicas de ensino: revisão integrativa. Revista Somma, 2016, p. 7483.

DELLA LUCIA, C.M., RODRIGUES, K.C.C., RODRIGUES, V.C.C., SANTOS, L.L.M., CARDOSO, L.M., MARTINO, H.S.D., FRANCHESCHINI, S.C.C, PINHEIRO-SANTANA, H.M. Diet quality and adequacy of nutrients in preschool children: Should rice fortified with micronutrients be included in school meals? Nutrients, 2016, 8 (5), 296.

EWUSIE, J.E., BEYENE, J., AHIADEKE, C., HAMID, J.S. Malnutrition in Pre-school Children across Different Geographic Areas and Socio-Demographic Groups in Ghana. Maternal and Child Health Journal, 2017, 21(4), p. 797-808.

GHASSEMI, A., KEIKHAEI, B. Effects of nutritional variables in children with iron deficiency anemia. International Journal of Pediatrics, 2014, 2 (3), p. 183-187.

GONÇALVES, H.V.B., CUNHA, D.T., STEDEFELDT, E., ROSSO, V.V. Family farming products on menus in school feeding: a partnership for promoting healthy eating. Ciência Rural, 2015, 45 (12), p. 2267-2273.

GOULART, R.M.M., BANDUK, M.L.S., TADDEI, J.A.A.C. Uma revista das ações de nutrição e do papel do nutricionista em creches. Revista de Nutrição, 2010, 23(4), p. 655665 .

HOUGHTON, L.A., BROWN, R.C., BEAUMONT, S., JENNINGS, S, BAILEY, K.B., HASZARD, J.J., ERHARDT, J., DANIELS, L., GIBSON, R.S. Micronutrient status differs among Maasai and Kamba preschoolers in a supplementary feeding programme in Kenya. Maternal and Child Nutrition, 2019, 15 (3).

KUPER, H., NYAPERA, V., EVANS, J., MUNYENDO, D., ZUURMOND, M., FRISON, S., MWENDA, V., OTIENO, D., KISIA, J. Malnutrition and Childhood Disability in Turkana, Kenya: Results from a Case-Control Study. PLoS ONE, 2015, 10 (12).

LIMA, D.B., FUJIMORI, E., BORGES, A.L., SILVA, M.M. Feeding in the two first years of life. Revista da Escola de Enfermagem da USP, 2011, 45, p. 1705-1709.

LONGO-SILVA, G., TOLONI, M.H., MENEZES, R.C.E., TEMTEO, T.L. MARIA ALICE A. OLIVEIRA, M.A.A., ASAKURA, L., COSTA, E.C., TADDEI, J.A.A.C. Intake of protein, calcium and sodium in public child day care centers. Revista Paulista de Pediatria, 2014, 32 (2), p. 193-199.

MARTINEZ, S.A., PINHO, F.N.L.G. Brasilian's school food policy: Social representations and marks from the past. Education Policy Analysis Archives, 2016, 24.

MELKU, M., ALENE, K.A., TEREFE, B., ENAWGAW, B., BIADGO, B. ABEBE, M., MUCHIE, K.F., KEBEDE,A., MELAK, T., MELKU, T. Anemia severity among children 
aged 6-59 months in Gondar town, Ethiopia: A community-based crosssectional study. Italian Journal of Pediatrics, 2018, 44 (1).

PEREIRA, I.F.D.S., ANDRADE, L.D.M.B., SPYRIDES, M.H.C., LYRA, C.D.O. Estado nutricional de menores de 5 anos de idade no Brasil: evidências da polarização epidemiológica nutricional. Ciência \& Saúde Coletiva, 2017, 22, p. 3341-3352.

PEREIRA, A.D.S., LANZILLOTTI, H.S., SOARES, E.D.A. Frequência à creche e estado nutricional de pré-escolares: uma revisão sistemática. Revista Paulista de Pediatria, 2010, $28,(4)$, p. 366-372.

RODRIGUES, V.C., MENDES, B.D., GOZZI, A., SANDRINI, F., SANTANA, R.G., MATIOLI, G. Deficiência de ferro, prevalência de anemia e fatores associados em crianças de creches públicas do oeste do Paraná, Brasil. Revista de Nutrição, 2011, 24, (3), p. 407-420.

SACHDEV, H.P.S., GERA, T. Preventing childhood anemia in India: Iron supplementation and beyond. European Journal of Clinical Nutrition 2013, 67 (5), p. 475-480.

SILVA, C.A.M., MARQUES, L.A., BONOMO, É., BEZERRA, O.M.P.A., CORRÊA, M.S.,PASSOS, L.S.F., DE SOUZA, A.A., BARROS, B.F., DE SOUZA, D.M.S., DOS REIS, J.A., DE ANDRADE, N.G. The Brazilian School Nutrition Program from the standpoint of students attending state schools in Minas Gerais. Ciência e Saúde Coletiva 2013, 18 (4), p. 963-969.

VALENCIA, Y.K.D., MEDINA, G.H.H., LAZO, J.G.L., VILCA, M.E.F., QUÍSPE, D.J.M., ORDOÑEZ, N.I.O., GALVEZ, S.J.Z. New Natural and Low Cost Product to Fight Anemia: Marketing and Profitability Study. International Engineering, Sciences and Technology Conference, 2019, p. 19-24.

VEIGA, G.R.S., FERREIRA, H.S., SAWAYA, A.L., CALADO, J., FLORÊNCIO, T.M. Dyslipidemia and undernutrition in children from impoverished areas of Maceio, state of Alagoas, Brazil. International Journal of Environmental Research and Public Health, 2010, 7 (12), p. 4139-4151.

VIEIRA, V.L., SOUZA, J.M.P., CERVATO-MANCUSO, A.M. Insegurança alimentar, vínculo mãe-filho e desnutrição infantil em área de alta vulnerabilidade social. Revista Brasileira Saúde Materna Infantil, 2010, 10, (2), p. 199-207. 


\title{
Declaração de Interesse
}

Os autores declaram não haver nenhum conflito de interesse

\section{Financiamento}

Programa de Amparo à Pesquisa da Faculdade Dinâmica do Vale Do Piranga - PROAPP/FADIP

\section{Agradecimentos}

\begin{abstract}
À Faculdade Dinâmica do Vale do Piranga pela concessão da Bolsa de Iniciação Cientifica no PIBIC e pelo Financiamento da Pesquisa PROAPP/FADIP
\end{abstract}

\section{Colaboração dos autores}

Moreira delineou o trabalho; Moreira, Teixeira e Silva coletaram os dados e escreveram o artigo; Moreira e Silva revisaram o trabalho final. 